Urologe $2016 \cdot 55: 615$

DOI 10.1007/s00120-016-0085-6

Online publiziert: 27. April 2016

(c) Springer-Verlag Berlin Heidelberg 2016

CrossMark

Maximilian Burger

Klinik für Urologie der Universität Regensburg, Caritas-Krankenhaus, St. Josef, Regensburg, Deutschland

\title{
Blasenerhaltende Therapie als Alternative zur Zystektomie
}

\section{Kommentar zur organerhaltenden Therapie des Harnblasenkarzinoms}

In ihrer Übersicht beleuchten Niedworok et al. organerhaltende Therapiestrategien beim muskelinvasiven Harnblasenkarzinom als mögliche Alternativen zur Zystektomie. Die Autoren haben auch die ältere Literatur dazu gründlich recherchiert. Sie beschreiben die einzelnen Ansätze klar und präzise, bewerten das Resultat ihrer Arbeit nachvollziehbar und haben zwei Botschaften: Erstens kann ein Erhalt der Blase auch beim muskelinvasiven Karzinom versucht werden. Und zweitens: die radikale Zystektomie ist die akzeptierte Therapie.

Von den endoluminalen Verfahren leuchtet die Lasertherapie noch am ehesten ein. Mit dem ja kaum überraschenden Phänomen, dass Rezidive an anderer als den gelaserten Stellen auftreten. Das invasive Karzinom ist ja ein Problem der ganzen Blase, was eigentlich gegen fokale Verfahren spricht. Also bietet sich als Alternative zur Zystektomie nur die trimodale Therapie an. Theoretisch zumindest.

Aber nur auf den ersten Blick sind die Ergebnisse mit der Zystektomie vergleichbar. Wie oft in unserem Metier lohnt die nähere Betrachtung. In einer der neueren und detaillierteren Arbei- ten, welche die Autoren unter der Referenz 30 auflisten, beträgt das karzinomspezifische 5-Jahres-Überleben eben mal $64 \%$ bei einer Rate an Salvagezystektomien von $29 \%$ [1].

Mich überzeugt das nicht. Und Herrn Niedworok und Kollegen nach Erarbeitung dieser gründlichen und guten Übersicht auch nicht. Sie konstatieren, dass die radikale Zystektomie die Therapie der Wahl bleibt. Das ist eine klare und richtige Botschaft.

\section{Korrespondenzadresse}

\section{Prof. Dr. M. Burger}

Klinik für Urologie der Universität Regensburg, Caritas-Krankenhaus, St. Josef Landshuter Straße 65, 93053 Regensburg, Deutschland

maximilian.burger@ukr.de

\section{Literatur}

1. Spiegel DY, Spiegel DY, Shipley WU, Heney NM, Kaufman DS, Niemierko A, Coen JJ, Skowronski RY, Paly JJ, McGovern FJ, Zietman AL (2012) Longterm outcomes of selective bladder preservation by combined-modality therapy for invasive bladder cancer: the MGH experience. Eur Urol 61(4):705-711 